\title{
Aplicación de campos estocásticos en problemas de geotecnia
}

\section{Application of stochastic fields in geotechnical problems}

\author{
Jorge Luis Palomino-Tamayo ${ }^{1}$, Armando Miguel Awruch ${ }^{2}$, Wilson Rodríguez-Calderón ${ }^{3}$ \\ ${ }^{1}$ Centro de Mecánica Aplicada y Computacional - CEMACOM, Facultad de Ingeniería Civil, Universidad Federal do Rio Grande \\ do Sul-UFRGS, Porto Alegre/RS, Brasil. Email: jorgetamayo@ufrgs.br \\ ${ }^{2}$ Centro de mecánica Aplicada y Computacional - CEMACOM, Facultad de Ingeniería Civil, Universidad Federal do Rio Grande \\ do Sul-UFRGS, Porto Alegre/RS, Brasil. Email: amawruch@ufrgs.br \\ ${ }^{3}$ Centro de mecánica Aplicada y Computacional - CEMACOM, Facultad de Ingeniería Civil, Universidad Federal do Rio Grande \\ do Sul-UFRGS, Porto Alegre, Brasil. Email: wilroca50@ hotmail.com
}

RECIBIDO: Enero 20, 2016. ACEPTADO: Marzo 1, 2017. VERSIÓN FINAL: Mayo 15, 2017.

\section{RESUMEN}

Este trabajo se enfoca en el análisis probabilístico de problemas de estabilidad de taludes y de asentamientos producidos en zapatas rígidas apoyadas en suelos deformables. Para este propósito son estudiados y combinados modelos matemáticos basados en el Método de los Elementos Finitos (MEF), método de Montecarlo (MC) y en el procedimiento de Subdivisión de Media Local (LAS). La metodología LAS se utiliza para generar campos estocásticos que representen apropiadamente las incertidumbres asociadas a las propiedades de los materiales. La utilización del MEF permite obtener la respuesta numérica del problema en términos de desplazamientos y tensiones. La plasticidad del suelo puede ser incluida a través de un algoritmo visco-plástico conjuntamente con el criterio de plastificación de Mohr-Coulomb. Los procedimientos LAS y MEF están incorporados dentro del marco de análisis del método de Montecarlo, donde cada análisis requiere la ejecución de varias simulaciones numéricas del problema en cuestión. Todo esto a fin de cuantificar una probabilidad de falla y el asentamiento más probable a ocurrir en el caso de problemas de cimentaciones. De los estudios realizados, se sugiere utilizar al menos 4000 y 500 simulaciones para los problemas del talud y zapata, respectivamente, a fin de obtener resultados estables y confiables. Los resultados obtenidos muestran que la probabilidad de falla para el talud estudiado está alrededor de 0.18 , mientras que el asentamiento más probable a ocurrir para un sistema suelo-zapata en condiciones de servicio está alrededor de $1.96 \mathrm{~cm}$.

PALABRAS CLAVE: Campos estocásticos, Elementos finitos, Análisis probabilístico, Geotecnia.

\begin{abstract}
This work focuses on the probabilistic analysis of slope stability and rigid shallow footing problems. For this purpose, mathematical models based on the Finite Element Method (FEM), Montecarlo (MC) method and Local Average Subdivision (LAS) procedure are studied. The LAS procedure is used to generate random fields, which properly represent the associated uncertainties in the properties of the materials. The FEM focuses on the numerical response of the problem in terms of displacements and stresses. The plasticity of the soil can be included via a visco-plastic algorithm beside a Mohr-Coulomb law. The LAS and MEF procedures are implemented in the framework of a MC analysis, where each MC execution requires several simulations of the problem at hand. This permits to quantify the failure probability of the system and report the most probable settlement to occur in the case of shallow foundations. After many executions of the numerical model, it is suggested that at least 4000 and 500 simulations are needed for the slope stability and shallow foundation problems, respectively, in order to obtain stable and reliable values. The obtained results show that the failure probability of the slope is relatively low and equal to 0.18 , while the expected settlement of a shallow rigid foundation is around $1.96 \mathrm{~cm}$.
\end{abstract}

KEYWORDS: Stochastic field, Finite element, Probabilistic analysis, Geotechnics.

Este artículo puede compartirse bajo la licencia CC BY-ND 4.0 y se referencia usando el siguiente formato: J. Palomino-Tamayo, A. Miguel Awruch, Wilson Rodríguez-Calderón, “Aplicación de campos estocásticos en problemas de geotecnia”, UIS Ingenierías, vol. 16, no. 2, pp. 185196, Julio-Diciembre 2017. Doi: https://doi.org/10.18273/revuin.v16n2-2017017 


\section{INTRODUCCIÓN}

Es importante cuantificar la confiabilidad de los sistemas estructurales frente a la posible aleatoriedad de las propiedades de los materiales. Esto último es relevante en el caso de los suelos y rocas debido a la alta variabilidad de sus propiedades principalmente en su estado natural. Por ejemplo, en una situación hipotética donde no existen restricciones económicas, es posible seleccionar algunas muestras de suelo de la zona del problema en estudio y determinar en laboratorio, las propiedades más importantes del material tales como su permeabilidad, compresibilidad, resistencia al corte, entre otras. Con esa información disponible es posible simular el problema numéricamente y tener un grado alto de confiabilidad en los resultados obtenidos. Sin embargo, en la práctica común se cuenta apenas con pocos datos de campo y laboratorio, siendo el enfoque usual considerar valores característicos para las propiedades de los materiales, conjuntamente con un factor de seguridad. De hecho, la mayoría de las normas de diseño siguen este enfoque.

En la literatura especializada existen varios enfoques para estudiar el problema de confiabilidad estructural, dentro de la grande diversidad de métodos, podemos citar el método llamado en la literatura inglesa de Random Finite Element Method (RFEM, Fenton y Griffith, 2008, Paiboon 2013, Bari 2012), el cual es utilizado en este trabajo. Este método combina la técnica MEF y MC con un generador de campos estocásticos basado en el procedimiento LAS. La metodología ha sido aplicada extensamente por otros investigadores para el estudio y análisis de problemas de estabilidad de taludes, cimientos, flujo a través de presas y licuefacción de suelos, entre otros. El método LAS es capaz de lanzar procesos aleatorios a nivel local en cada elemento finito que compone la malla. Las rutinas de computador disponibles en Fenton y Griffith (2008) son utilizados en este trabajo para simular el comportamiento probabilístico de un talud y de un sistema suelo-zapata.

Existen otras metodologías para el estudio de confiabilidad estructural o riesgo, dentro de estas técnicas se puede mencionar la técnica llamada en la literatura inglesa de First Order Realiability Method (FORM), simulación directa de Montecarlo y simulación de Montecarlo con muestreo por importancia adaptativo (MCIS), entre otras. Cavalcante et al. (2005) estudio el análisis de riesgo en taludes utilizando el método de Montecarlo, PEM (Point Estimate Method) y la teoría de conjuntos difusos. Bressani y Costa (2005) estudiaron probabilísticamente la estabilidad de un talud coluvionar de basalto siguiendo una metodología que incluye un análisis determinista inicial, caracterización de las variables de entrada (simulación de las incertidumbres y correlación) y aplicación de método probabilístico con método de Montecarlo. Temas probabilísticos también han sido destinados a la estimación del daño sísmico en puentes (Lamus et al. 2012).

\section{MÉTODOS}

\subsection{Modelamiento estocástico}

En la Fig. 1 se ilustran las propiedades estadísticas de una arcilla no drenada, la cual es caracterizada por su resistencia al corte no drenada, $X=c_{u}$. La Fig. 1(a) muestra la variación de esta cohesión $c_{u}$ con la profundidad de un depósito de suelo uniforme. En el caso de un proyecto convencional, se consideraría apenas la media, $\mu$, de esta propiedad en el análisis numérico o algún otro valor característico sugerido por alguna norma de diseño. Sin embargo, en un proyecto estocástico todas las propiedades de los materiales o por lo menos las más importantes deben ser representadas a través de su media y desviación estándar como se ilustra en la Fig. 1(b). En este último caso, la variabilidad de las propiedades es expresada en términos de las funciones de densidad de probabilidad (pdf), las cuales son precisamente caracterizadas por la media $\mu$ y una desviación estándar normalizada, $\sigma$. Estas consideraciones definen el llamado coeficiente de variación $V$ mostrado en la Ec.1.

$$
V=\frac{\sigma}{\mu}
$$

La función de densidad de probabilidad mostrada en la Fig. 1 (b) sigue una distribución normal, aunque otras distribuciones del tipo log-normal, exponencial, beta, entre otras, podrían ser utilizadas a fin de mejorar la representación espacial de las propiedades. Los conceptos de media y desviación estándar son también utilizados para describir la variación de los parámetros de resistencia con la profundidad $z$ de un depósito de suelo. Por ejemplo, Lumb (1966) consideró una tendencia lineal para una arcilla no drenada de la siguiente manera:

$$
c_{u}=\mu+\sigma u
$$

donde $c_{u}$ es la resistencia al corte no drenada y:

$$
\begin{aligned}
& \mu=\mu(z)=\propto_{u}+\beta_{u} z \\
& \sigma=\sigma(z)=\propto_{\sigma}+\beta_{\sigma} z
\end{aligned}
$$

con $u$ siendo una variable aleatoria normalizada con media cero y desviación estándar unitaria, la cual varía de acuerdo con el tipo de función de densidad de 
probabilidad seleccionada. Para una distribución normal, esta función se define por la Ec. 5. Donde $\propto_{u}, \propto_{\sigma}, \beta_{u}$ y $\beta_{\sigma}$ en las ecuaciones 3 y 4 representan parámetros que definen la variación de la media y desviación estándar, respectivamente, de la propiedad en cuestión con la profundidad del suelo.

$$
g(u)=\frac{1}{\sqrt{2 \pi}} \exp \left(-\frac{1}{2} u^{2}\right)
$$

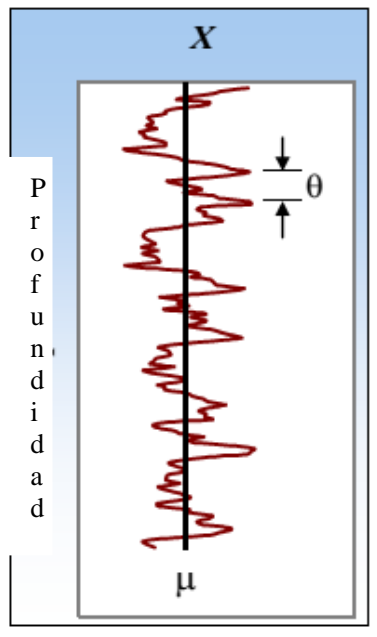

a) $\quad c_{u}$ como función de la profundidad $\mathrm{z}$

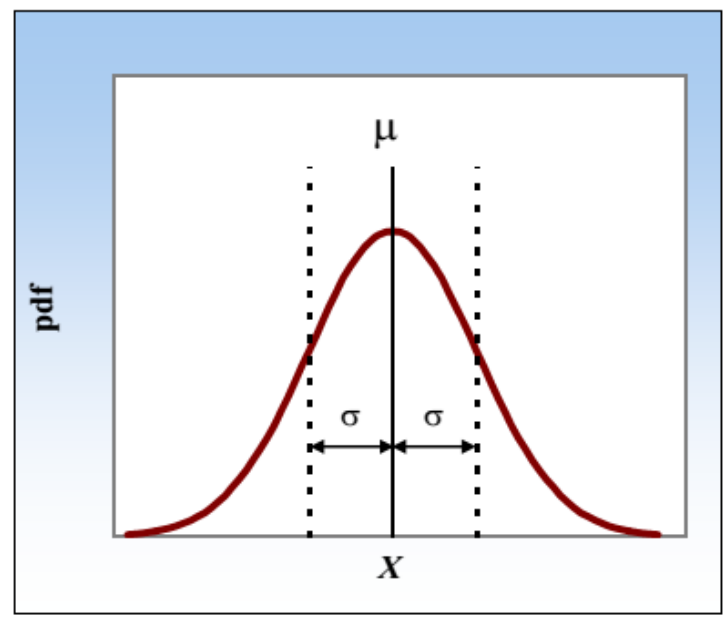

b) Función de densidad de probabilidad de $c_{u}$

Figura 1. Representación de la variabilidad de la resistencia al corte no drenada $c_{u}$ del suelo. Fuente. Elaboración propia.

En la Ec. (3), para que la media $c_{u}$ sea constante con la profundidad (como se representa por la línea negra en la Fig. 1 (a)), se requiere que $\beta u$ sea zero, mientras que $\alpha_{u}=$ $\alpha_{\sigma}=0$ permite que $\mu$ y $\sigma$ se incrementen linealmente desde valores nulos en la superficie del suelo (en $z=0$ ). En este caso, el coeficiente $V=\beta_{\sigma} / \beta_{\mu}$ de la Ec. 1 adopta un valor constante a lo largo de la profundidad.

De esta manera, las ecuaciones (1)-(5) definen parcialmente la variabilidad de las propiedades en el dominio espacial, pero también se requiere de la definición de un parámetro estadístico adicional, $\theta$, que define el grado de correlación espacial entre las zonas adyacentes (Vanmarcke, 1983, Vanmarcke 1977). Precisamente, la Fig. 1 (a) muestra que $\theta$ depende de la distancia entre estas zonas. Por lo tanto, para un material débilmente correlacionado, la distancia de correlación $\theta$ es menor, mientras que para materiales altamente correlacionados, el valor de $\theta$ aumenta.

Una vez que las variables las estadísticas $(\mu, \sigma, \theta)$ de una propiedad de un tipo de suelo son conocidas, una simulación numérica del campo de distribución de esta propiedad puede ser generada utilizando el método de Subdivisión de Media Local (LAS) (Fenton y Vanmarcke, 1990; Fenton 1990). Esta metodología genera medias locales correlacionadas $Z$ de acuerdo con una función de densidad de probabilidad Gaussiana (con media cero y varianza unitaria) y una función de correlación espacial $\rho$. Para un campo estocástico isotrópico, la función de correlación de Gauss-Markov se define de acuerdo con la Ec. (6).

$$
\rho(\tau)=\exp \left(-\frac{2}{\theta}|\tau|\right)
$$

donde $\tau$ es un vector de desfase. El campo estocástico así generado es entonces transformado de un campo Gaussiano para otro definido por la función de densidad de probabilidad (pdf), utilizando las expresiones de $\mu(z)$ y $\sigma(z)$ de las Ecuaciones (3)-(4), respectivamente. Para una distribución normal, la transformación se define de la siguiente manera:

$$
c_{u}(x)=\mu(z)+\sigma(z) Z(x)
$$

donde para problemas planos $2 \mathrm{D}, Z(x)$ representa la media local de una celda de un campo estocástico con centroide en $x=(x, z)^{\mathrm{T}}$. El método LAS en su versión actual utiliza celdas cuadradas para generar el campo estocástico, esto significa que el proceso de subdivisión se realiza a partir de un dominio externo cuadrado pasando por una discretización sucesiva hasta llegar a una versión final, formada por varias celdas cuadradas menores como se muestra en la Fig. 2. Cada una de estas 
celdas está asociada a una única media local, la cual esta correlacionada con las propiedades de las celdas adyacentes.

Para un grupo de datos estadísticos $(\mu, \sigma, \theta)$, existe un número infinito de campos estocásticos posibles a ser

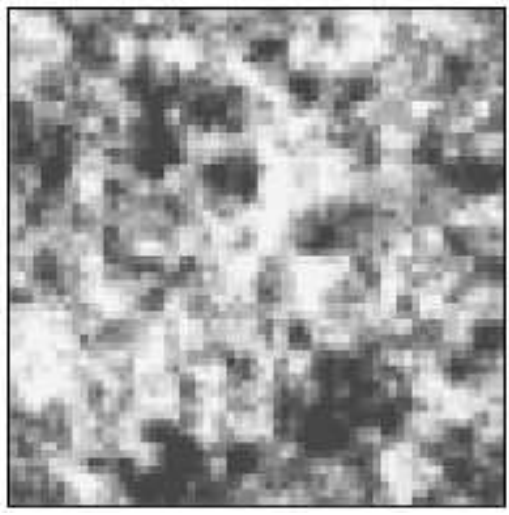

a) $\theta / D=0.1$ generados. Aunque estadísticamente similares, cada campo representa una distribución espacial diferente de la propiedad del material en cuestión.

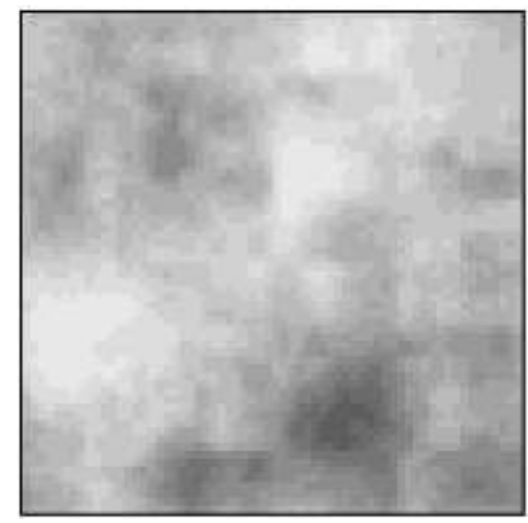

b) $\theta / D=1$

Figura 2. Campos estocásticos bidimensionales de la variable $X$ generados con LAS, donde $D$ representa la dimensión del dominio. Fuente. Elaboración propia.

Por lo tanto, un análisis estocástico implica una serie de análisis numéricos con MEF, las cuales son llamadas de simulaciones y que están inmersas dentro del análisis de Montecarlo. Los resultados obtenidos de las simulaciones conllevan a un análisis de confiabilidad o riesgo, más que a la determinación de un único factor de seguridad. Es decir, los resultados se expresan como funciones de densidad de probabilidad (pdf), permitiendo establecer la probabilidad de falla de una estructura en situaciones de colapso o en la obtención del valor más probable de una variable de interés como ocurre en el cálculo del asentamiento de una zapata. Cuanto mayor es el número de simulaciones más confiable es el resultado obtenido, sin embargo, en la práctica no es posible realizar infinitos análisis numéricos por diferentes razones. Por ejemplo, un número grande de simulaciones conllevará a una demanda considerable de tiempo de cómputo, hecho que todavía puede ser agravado a través de la utilización de una malla de EF bastante refinada. De esta manera, es deseable establecer un número mínimo de simulaciones a fin de obtener resultados confiables, estables y asegurar un tiempo de cómputo adecuado.

\subsection{Algoritmo RFEM}

Los parámetros estadísticos $\mu, \sigma, \theta$ de una variable de estado (por ejemplo, la resistencia no drenada al corte de un suelo) pueden ser considerados a ser definidos a nivel puntual. Sin embargo, las estadísticas a ese nivel de resolución son obviamente imposibles de medir en la práctica, esto genera entonces incertidumbres asociadas con la variabilidad del suelo. Lo anterior puede ser corregido utilizando una media local que lleve en consideración el tamaño de la muestra, en este contexto el método LAS actúa, mediante la generación de campos estocásticos para las propiedades de interés del suelo. LAS está acoplado al MEF y al método MC, para así definir el método RFEM. Es decir, a cada elemento o celda generada se le atribuye un único valor estocástico de la propiedad, la cual permanece constante en el dominio de la celda. De esta manera, la muestra del suelo es representada por el tamaño de cada celda o elemento finito utilizado para discretizar el dominio. $\mathrm{Si}$ la distribución del punto en cuestión es normal, el proceso de subdivisión de media local aplicada al dominio en estudio, resulta en una varianza reducida de la propiedad del material, pero con su media inalterada. Sin embargo, en una distribución log-normal, tanto la media y la desviación estándar se reducen durante el proceso, pues la media de una distribución log-normal depende de la media y varianza de la distribución normal asociada. Por lo tanto, una discretización grosera del problema con mayores tamaños para los elementos, tendrá una mayor influencia del proceso de media local. Estos ajustes en los puntos estadísticos son considerados dentro del algoritmo del método RFEM.

\section{RESULTADOS}

\subsection{Estudio probabilístico de la estabilidad de un talud}

En este caso, se estudia la probabilidad de falla del talud mostrado en la Fig. 3 con la metodología RFEM. La malla de elementos finitos utilizada en el modelo numérico también es mostrada en esta figura. El talud 
presenta una pendiente 2: 1 con altura $H=0,5 \mathrm{~m} \mathrm{y}$ profundidad $D=0.75 \mathrm{~m}$. Las condiciones de contorno son: base fija y apoyos deslizantes en los contornos laterales. Cada elemento finito dentro de la malla es un cuadrado de $0.05 \mathrm{~m}$ de lado. La discretización a lo largo de la dirección horizontal está compuesta por 60 elementos (3 m) y a lo largo del mayor lado vertical por 15 elementos $(0.75 \mathrm{~m})$. En total se utilizan 610 elementos finitos planos de ocho nodos con la hipótesis de deformación plana. Es importante señalar que las dimensiones del talud son apenas representativas y pueden corresponder a un modelo en escala. La distancia de correlación del talud es parametrizada con $\Theta=\theta / H$, donde $\theta$ es la distancia de correlación, todo esto con la finalidad de extender la validez de los resultados para otras geometrías con igual valor de $H / D$.

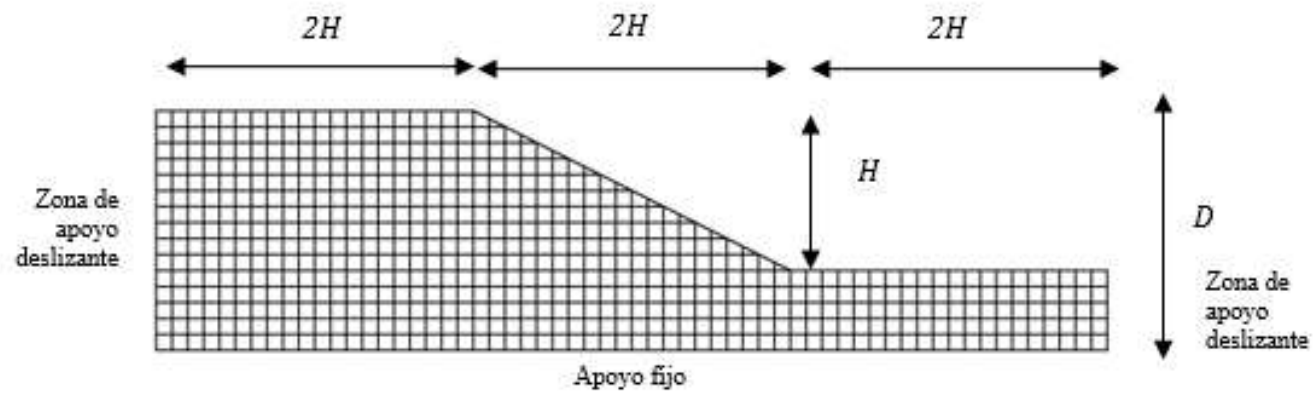

Figura 3. Malla de elementos finitos utilizada. Fuente. Elaboración propia.

La distancia de correlación se supone igual en la dirección vertical y horizontal con $\theta_{\ln E}=\theta_{\ln \gamma s}=\theta_{\operatorname{lncu}}=1$ $\mathrm{m}$, donde $\theta l_{n E}, \theta_{l n \gamma s}$ y $\theta_{\text {lncu }}$ son las distancias de correlación para el módulo de elasticidad, peso específico y cohesión no drenada del suelo, respectivamente. La media y la desviación estándar de $c_{u}$ (cohesión no drenada del suelo) son $\mu_{c u}=2 \mathrm{kPa} \mathrm{y} \sigma_{c u}=0.5 \mathrm{kPa}$, respectivamente $(V=0.25)$, la media y la desviación estándar para $\gamma_{s}$ (peso específico del suelo) son $\mu_{\gamma s}=20 \mathrm{kN} / \mathrm{m}^{3}$ y $\sigma_{\gamma s}=2 \mathrm{kN} / \mathrm{m}^{3}$, respectivamente y la media y desviación estándar para $E$ (módulo de elasticidad) son $\mu_{E}=100000 \mathrm{kPa} \mathrm{y} \sigma_{E}=$ $50000 \mathrm{kPa}$, respectivamente. Otras variables tales como el ángulo de fricción $\left(\varnothing=0^{\circ}\right)$, el ángulo de dilatancia $(\psi$ $\left.=0^{\circ}\right) \mathrm{y}$ el coeficiente de Poisson $(v=0)$ son consideradas variables deterministas y, por lo tanto, constantes durante el análisis numérico. La distancia de correlación parametrizada es $\Theta=\theta / H=2$, mientras que otras variables son: $H / D=0.67, V_{E}=\sigma_{E} / \mu_{E}=0.5, V_{c u}=\sigma_{c u}$ / $\mu_{c u}=0.25, V_{\gamma s}=\sigma_{\gamma s} / \mu_{\gamma s}=0.1, \mu_{N s}=\mu_{c u} /\left(\mu_{\gamma s} H\right)=0.2$.

El nivel de correlación entre parámetros de resistencia, tales como $c$ y $\emptyset$ está pobremente documentado en la literatura, además de no existir un consenso sobre sus valores entre los investigadores. Sin embargo, valores en el rango de -0.24 a -0.70 ha sido reportado en la literatura (Chok, 2008). El signo negativo se refiere a una correlación totalmente negativa (osea un mayor valor de $c$ corresponde con un menor valor de $\emptyset$ y viceversa). Sin embargo, en este trabajo se supone una correlación totalmente positiva para $c$ y $\varnothing$ con un coeficiente de correlación igual a 0.4 . La correlación cruzada entre c y $\emptyset$ se considera utilizando la aproximación de descomposición de matriz de covariancia, la cual está bien documentada en el trabajo de Fenton y Griffiths (2008).

El presente análisis numérico implica la aplicación de cargas de gravedad y el monitoreo de las tensiones en los puntos de integración de cada elemento finito. Las fuerzas generadas por el peso propio del depósito son calculadas por el procedimiento conocido en la literatura inglesa como turn-on procedure (Smith et al., 2014). Este procedimiento genera tensiones normales y de corte en los puntos de integración dentro de la malla, los cuales serán posteriormente utilizadas para evaluar el criterio de plastificación de Mohr-Coulomb, y de esta manera tener en cuenta la no linealidad del material.

En primer lugar, un análisis determinista es realizado considerando apenas las propiedades medias del suelo. De esta manera, se puede obtener factores de seguridad reduciendo proporcionalmente los valores de los parámetros de resistencia (cohesión y ángulo de fricción) hasta encontrar la condición de falla del talud. Esta condición de falla se define numéricamente cuando el algoritmo no converge después de 500 iteraciones (valor sugerido por Fenton y Griffith, 2008). Los resultados obtenidos así, indicaron un factor de seguridad de 1.30.

En una segunda fase, un análisis probabilístico o estocástico es realizado considerando diferentes campos estocásticos para cada una de las propiedades de interés del suelo. Los valores generados de esta manera son transformados a un campo logarítmico (valores positivos) para ser finalmente mapeados a la malla de elementos finitos (Fenton y Griffiths 2008). Durante las simulaciones numéricas, el código de EF se ejecuta en 
modo de detección falla. Es decir, las simulaciones que requieren más de 500 iteraciones para convergir o aquellas que no convergen caracterizan las poblaciones de falla. Después de un número suficiente de simulaciones (alrededor de 20000), la probabilidad de falla es determinada como la división entre el número de simulaciones que indican falla y el número total de simulaciones.

El número de simulaciones necesarias dentro de un análisis de Montecarlo para obtener una salida estable de los resultados depende en gran medida de la variabilidad de los datos de entrada. La Fig. 4 muestra la probabilidad de falla para el presente talud como función del número de simulaciones. Esta probabilidad es de aproximadamente de 0,18 y se estabiliza para al menos 4000 simulaciones. Por lo tanto, se sugiere ese valor mínimo para el análisis probabilístico de taludes con geometría similar a aquella mostrada en la Fig. 3. Es importante destacar que cada punto de la curva mostrada en la Fig. 4 corresponde a un análisis de MC con un número predeterminado de simulaciones.

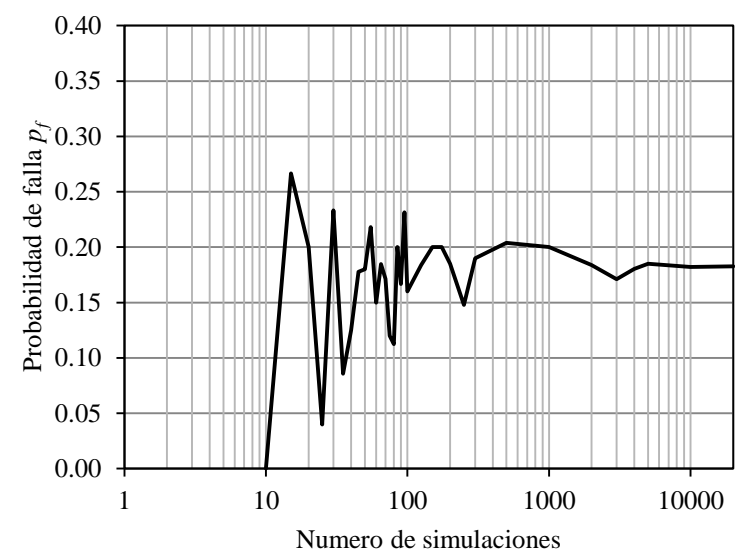

Figura 4. Estabilización de la probabilidad de falla con el número de simulaciones. Fuente. Elaboración propia.

Como ilustración, en la Fig. 5 se presentan en escala de grises los diferentes campos estocásticos generados para la cohesión del suelo de acuerdo con la metodología LAS en diferentes simulaciones. Las regiones más oscuras y claras representan mayores y menores valores asignados para la cohesión, respectivamente. Después de la aplicación del peso propio sobre el talud, las configuraciones deformadas obtenidas para las mallas mostradas en la Fig. 5 son mostradas en la Fig. 6. Como se puede apreciar, para el grupo de simulaciones mostradas, apenas la simulación No 1000 representa una condición de falla del talud después de no lograr convergir en 500 iteraciones. Otro detalle a destacar es que no necesariamente la superficie de ruptura del talud es circular como se sugiere en otras metodologías (por ejemplo, aquellas basadas en métodos de equilibrio limite o dovelas). Precisamente esto constituye una ventaja de la presente metodología que no supone un plano de falla a priori. En la Fig. 7 se muestran los campos de velocidades de deformación del talud. Como se puede apreciar, la simulación $\mathrm{N}^{\mathrm{o}} 1000$ presenta inicialmente una superficie de falla casi circular la cual se extiende hasta la superficie horizontal superior del talud.

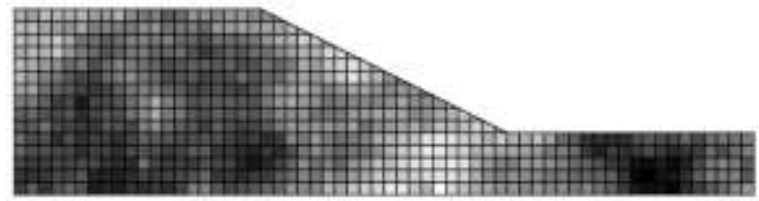

a) Malla de EF correspondiente a la simulación $\mathrm{N}^{\mathrm{o}} 1$

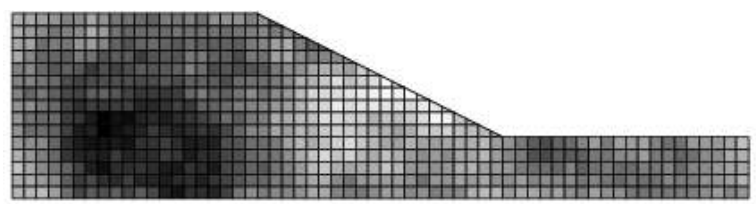

b) Malla de EF correspondiente a la simulación $\mathrm{N}^{\circ} 100$

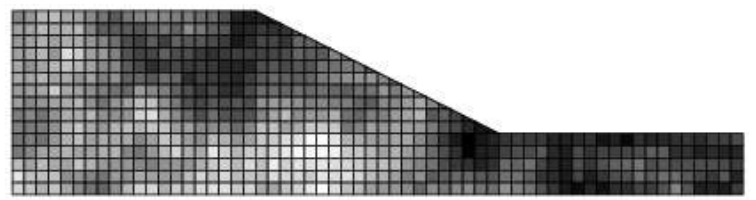

c) Malla de EF correspondiente a la simulación $\mathrm{N}^{\circ} 300$

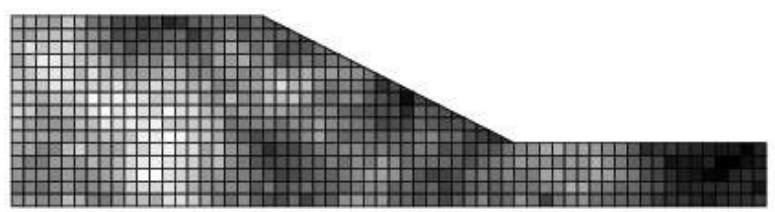

d) Malla de EF correspondiente a la simulación $\mathrm{N}^{\circ} 500$

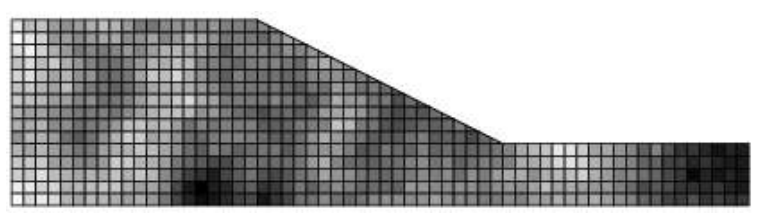

e) Malla de EF correspondiente a la simulación $\mathrm{N}^{\circ} 800$

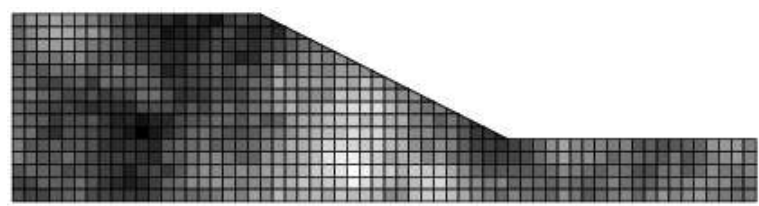

f) Malla de EF correspondiente a la simulación $\mathrm{N}^{\mathrm{o}} 1000$

Figura 5. Generación de campos estocásticos para la cohesión. Fuente. Elaboración propia. 


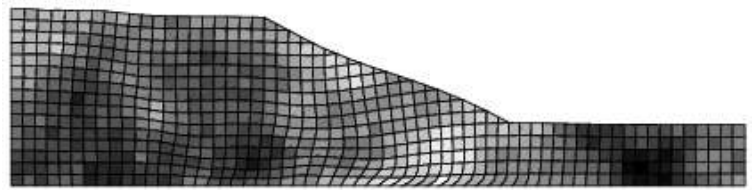

a) Configuración deformada correspondiente a la simulación $\mathrm{N}^{\mathrm{o}} 1$ (sin falla)

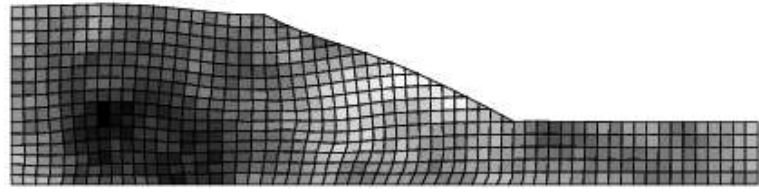

b) Configuración deformada correspondiente a la simulación $\mathrm{N}^{\circ} 100$ (sin falla)

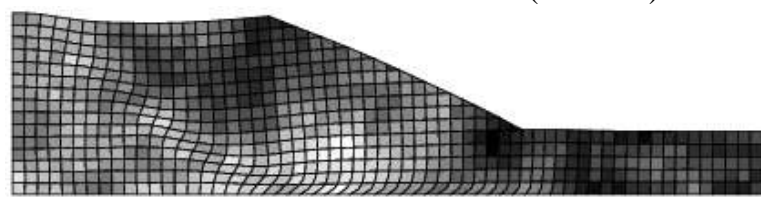

c) Configuración deformada correspondiente a la simulación $\mathrm{N}^{\circ} 300$ (sin falla)

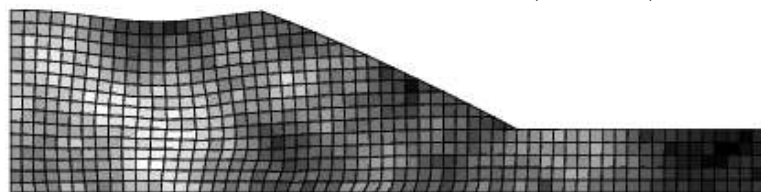

d) Configuración deformada correspondiente a la simulación No $^{0} 500$ (sin falla)

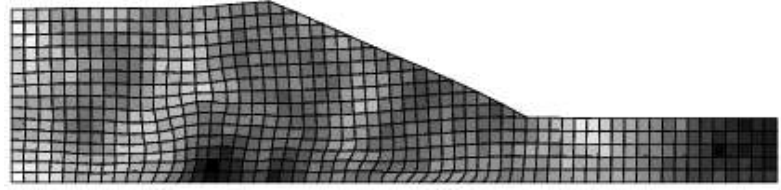

e) Configuración deformada correspondiente a la simulación $\mathrm{N}^{\circ} 800$ (sin falla)

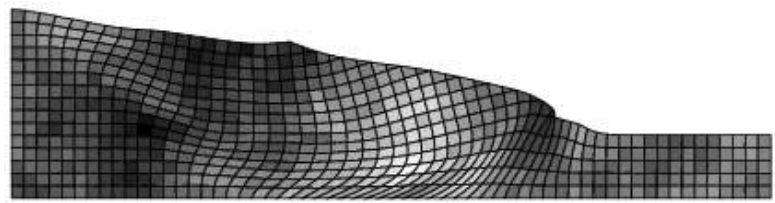

f) Configuración deformada correspondiente a la simulación $\mathrm{N}^{\circ} 1000$ (falla)

Figura 6. Configuraciones deformadas del talud. Fuente. Elaboración propia.

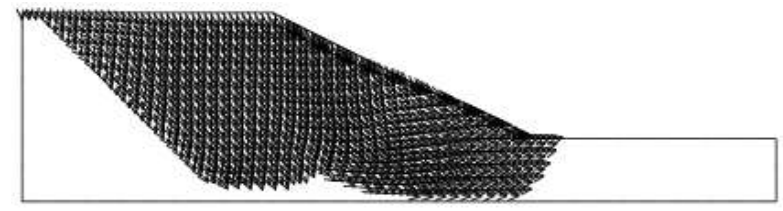

a) Simulación $\mathrm{N}^{\mathrm{o}} 1$

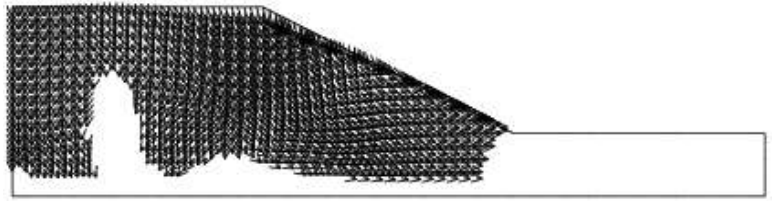

b) Simulación $\mathrm{N}^{\mathrm{o}} 100$

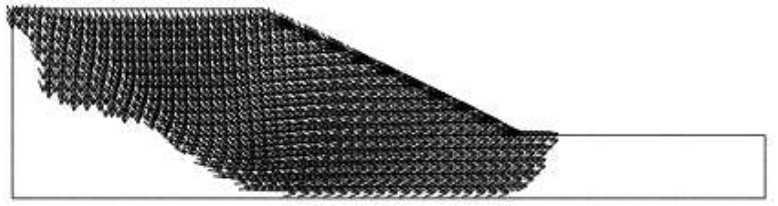

c) Simulación $N^{\circ} 300$

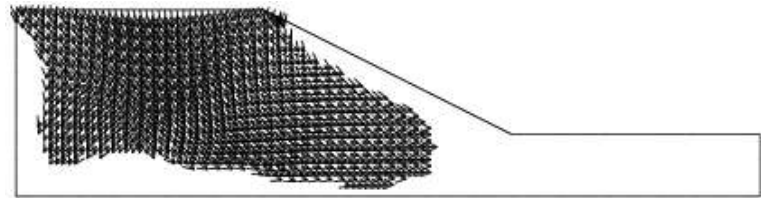

d) Simulación $\mathrm{N}^{\circ} 500$

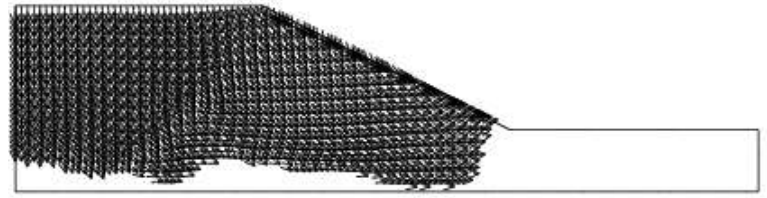

e) Simulación $N^{\circ} 800$

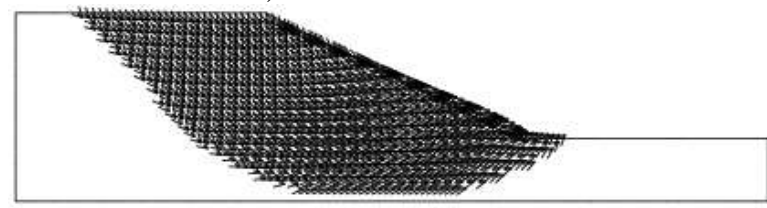

f) Simulación $N^{\circ} 1000$ (falla)

Figura 7. Campo de velocidad de deformación. Fuente. Elaboración propia. 


\subsection{Estudio probabilístico de asentamientos producidos por una zapata rígida en el suelo reformable.}

Como segundo ejemplo, se realiza un estudio probabilístico para el cálculo del asentamiento producido por la carga transmitida por una zapata cuadrada rígida apoyada en un suelo deformable. La diferencia con el ejemplo anterior, radica en la simulación numérica tridimensional del problema. El módulo de elasticidad del suelo es la única variable aleatoria a considerar, la cual será modelada mediante un campo estocástico tridimensional, otras propiedades se consideran deterministas. El suelo se supone isotrópico elástico con una estructura de correlación igual en la dirección vertical cuanto en la dirección horizontal apenas por simplicidad. Sin embargo, se sabe que existe una mayor correlación en la dirección horizontal debido a la historia estratigráfica del suelo. La zapata está apoyada sobre una capa de suelo deformable, y ésta su vez se apoya en una capa rígida (condición de apoyo fijo en la base de la malla de elementos finitos) como se muestra en la Fig. 8. El depósito del suelo es discretizado utilizando elementos hexahedricos de ocho nudos, 64 elementos en cada dirección horizontal y 32 elementos en la dirección vertical, totalizando 131072 elementos finitos.

Cada elemento es cubico con $0.15 \mathrm{~m}$ de lado, definiendo un depósito con dimensiones en el plano horizontal (XY) de $9.6 \mathrm{~m}$ x $9.6 \mathrm{~m}$ y una profundidad de $4.8 \mathrm{~m}$. Se observa que las unidades de distancia son arbitrarias desde que se utilicen unidades consistentes de presión y fuerza. La carga de servicio aplicada en cada nudo de la malla en la región bajo la zapata es de 1200 kN (Ver Fig. 8).
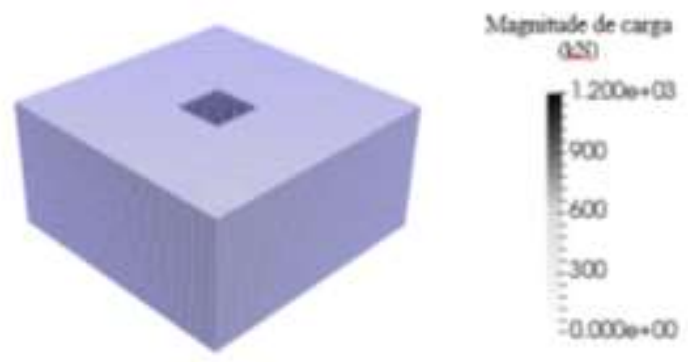

Figura 8. Malla de elementos finitos del sistema suelo-zapata y valor de la carga aplicada en kN. Fuente. Elaboración propia.

El análisis de elementos finitos utiliza un solucionador de sistema de ecuaciones del tipo gradiente conjugado con pre-condicionador lo que evita el montaje de la matriz de rigidez del sistema. Las caras verticales de la malla están restringidas para moverse horizontalmente, pero son libres de desplazarse verticalmente, mientras que los nudos de la base se fijan en todas las direcciones simulando una condición rígida. Se supone que la zapata es rígida (sin rotación) y está adherida al suelo elástico de cimentación (sin deslizamiento). El asentamiento depende de la magnitud de la carga aplicada y, por lo tanto, los resultados se pueden expresar de forma paramétrica a fin de extender su aplicabilidad a otros sistemas suelo-zapata con diferentes dimensiones, pero con relaciones de $B / H$ equivalentes, donde $B$ es ancho de la zapata y $H$ es la espesura del depósito de suelo. Por ejemplo, el asentamiento de una zapata cuadrada de dimensiones $B=4.0 \mathrm{~m}$ apoyada en una capa de suelo de espesor $H^{\prime}=20 \mathrm{~m}$ con $P^{\prime}=1000 \mathrm{kN}$ y módulo de elasticidad $E^{\prime}=60 \mathrm{kN} / \mathrm{m}^{2}$ corresponde a $5 / 6$ veces el asentamiento de una zapata de ancho $B^{\prime}=0.2 \mathrm{~m}$ apoyada en una capa de suelo de espesor $H=1 \mathrm{~m}$ con $P=1 \mathrm{kN}$ y módulo elástico $E=1 \mathrm{kN} / \mathrm{m}^{2}$. El factor de escala del primero para el segundo sistema se define por el factor $\left(P^{\prime} / P\right)\left(E / E^{\prime}\right)\left(B / B^{\prime}\right)$ siempre que $B^{\prime} / H^{\prime}=B / H$. Si $B / H$ no es constante, otro análisis determinista debe ser realizado a fin de determinar el nuevo factor de escala. En el presente caso, se utilizaron los siguientes valores: $B=1.8 \mathrm{~m}, H=4.8$ $\mathrm{m}, \theta_{l n E}=1 \mathrm{~m}, \mu_{E}=20000 \mathrm{kPa}, \sigma_{E}=10000 \mathrm{kPa}, \mathrm{B} / \mathrm{H}=$ 0.375 y $\sigma_{E} / \mu_{E}=0.5$.

El suelo tiene dos propiedades de interés, el módulo de elasticidad $\mathrm{E}(x)$ y el coeficiente de Poisson $\mathrm{v}(x)$, los cuales dependen de la posición espacial $x$. En este estudio se considerará apenas la aleatoriedad del módulo de elasticidad. El coeficiente de Poisson presenta una variabilidad de segunda importancia según Fenton y Griffiths (2008). La Fig. 9 muestra el campo estocástico tridimensional generado para el módulo de elasticidad en una simulación típica. Para este propósito, el algoritmo tridimensional del proceso LAS es utilizado. Las rutinas de cómputo para este caso son también encontradas en el trabajo de Fenton y Griffith (2008). Por lo tanto, para mayores detalles sobre el algoritmo numérico y el código fuente, el lector es direccionado para dicha referencia.

En primer lugar, se realizan varios análisis de Montecarlo con diferentes números de simulaciones hasta llegar a un valor máximo de 5000 simulaciones. En la Fig. 10 se muestra la variación del asentamiento máximo bajo la zapata conforme el número de simulaciones aumenta. Como se puede observar, el valor del asentamiento se estabiliza aproximadamente después de 500 simulaciones. De otra manera, en la Fig. 11 se muestra la variación de la desviación estándar del asentamiento con el número de simulaciones, es decir, en cada ejecución de un ciclo de Montecarlo, se calcula la media y desviación estándar de esta variable. Se puede observar que la desviación estándar se estabiliza también después de 500 simulaciones. Por lo tanto, se sugiere considerar al menos 500 simulaciones para el análisis probabilístico de este tipo de problemas.

Finalmente, en la Fig. 12 se muestra el histograma correspondiente al asentamiento máximo producido bajo 
la zapata obtenido para un análisis de MC con 5000 simulaciones. Se puede observar que la tendencia del asentamiento es seguir la forma de una distribución normal o log-normal. El asentamiento con valor igual a $1.957 \mathrm{~cm}$ tiene una frecuencia de 500 veces, resultando ser el valor más probable. Es importante destacar que varias normas de cimentaciones limitan el asentamiento máximo diferencial en servicio alrededor de $2.5 \mathrm{~cm}$.

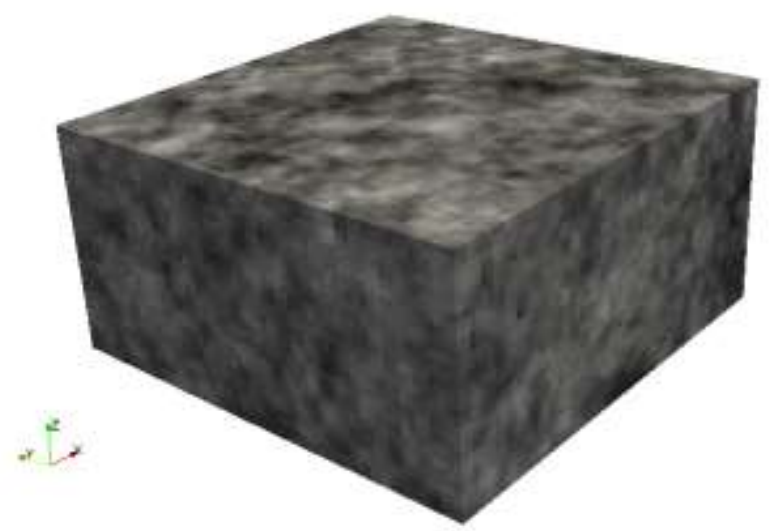

Figura 9: Campo estocástico para el módulo de elasticidad del suelo generado con la versión tridimensional del método LAS. Fuente: Elaboración propia.

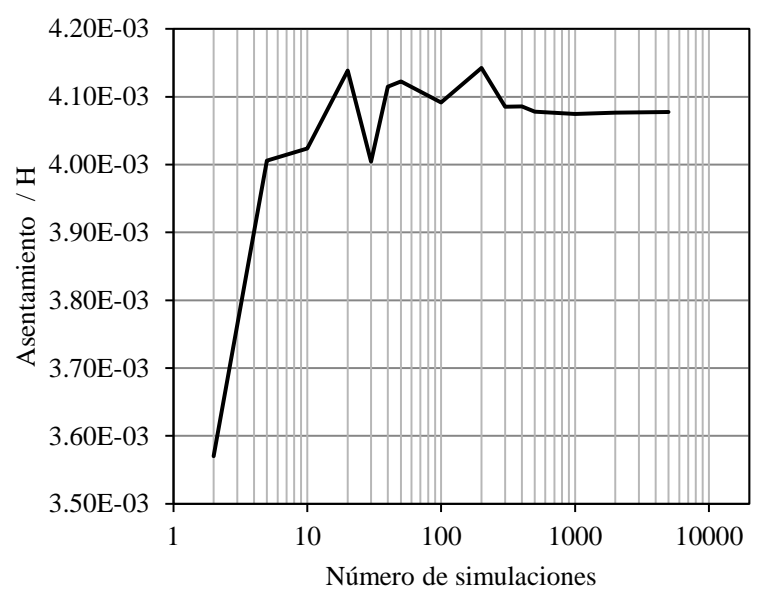

Figura 10. Variación del asentamiento máximo con el número de simulaciones. Fuente. Elaboración propia.

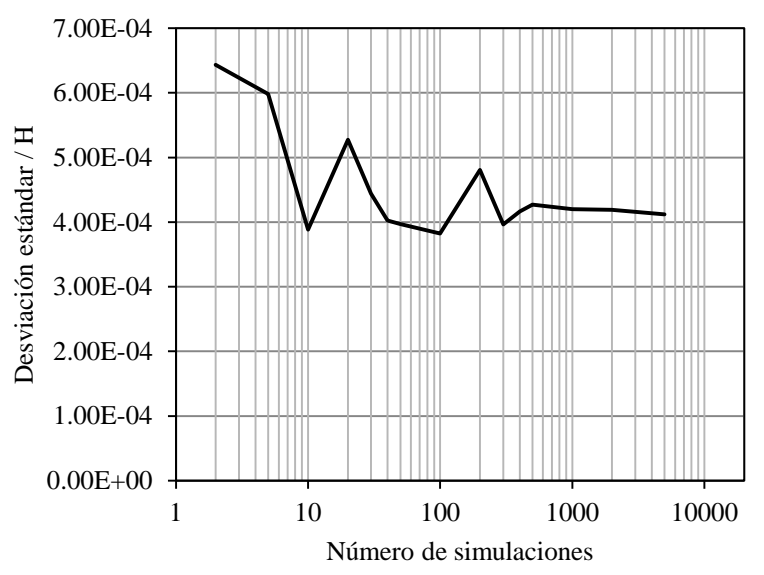

Figura 11: Variación de la desviación estándar del asentamiento máximo con el número de simulaciones. Fuente. Elaboración propia.

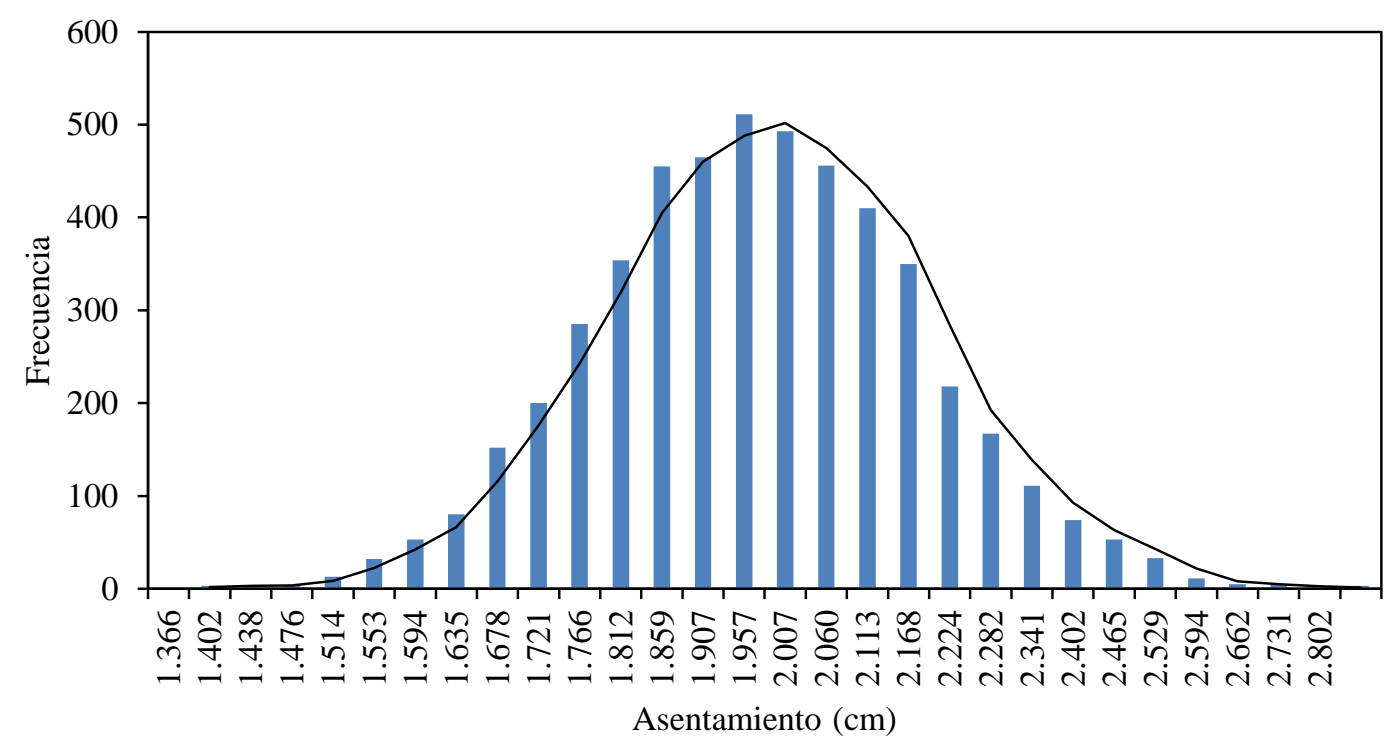

Figura 12. Histograma de asentamientos después de 5000 simulaciones. Fuente. Elaboración propia. 


\section{CONCLUSIONES}

- La eficiencia del modelo numérico en el cálculo de probabilidades de falla o determinación de asentamientos probables está asociada al esfuerzo computacional de un análisis de Montecarlo, la cual a su vez depende del número de simulaciones. Cuanto mayor es el número de simulaciones mayor será la confiabilidad en los resultados obtenidos, aunque esto también estaría asociado a un incremento en el tiempo de cómputo, llegando a ser prohibitivo en algunos casos, principalmente cuando son utilizados computadores personales para la simulación. De esta manera, es deseable definir un número de simulaciones mínimo que consiga satisfacer simultáneamente la estabilidad de los resultados con el menor esfuerzo de cómputo. Para el ejemplo del talud, la probabilidad de falla por peso propio fue de 0.18, valor que se estabiliza después de 4000 simulaciones. Ya en el caso del cálculo del asentamiento de una zapata rígida apoyada en un depósito de suelo deformable, el valor más probable esta alrededor de 1.96 $\mathrm{cm}$ después de 500 simulaciones. Como una primera tentativa para análisis estocásticos de estos problemas se sugiere utilizar dichos valores.

- El análisis determinista del talud sugiere un factor de seguridad alrededor de 1.3 en condiciones de peso propio. Esto significa que la probabilidad de falla asociada seria cero, pues el factor de seguridad es mayor que uno. Contrariamente, el análisis estocástico predice una probabilidad de falla igual a 0.18 para el mismo talud. Esto muestra la importancia de realizar un análisis que lleve en consideración las incertidumbres de los materiales. Queda pendiente la extensión del presente estudio para la consideración de las incertidumbres asociadas a las cargas aplicadas en el sistema.

- La parte más compleja del algoritmo RFEM corresponde al procedimiento LAS para la generación del campo estocástico de la variable aleatoria de interés. La metodología LAS utilizada aquí se mostró robusta numéricamente en lo que se refiere a tiempos de cómputo para un computador personal. Las rutinas y programas obtenidos del trabajo de Fenton y Griffiths (2008) pueden ser modificados o incluidos directamente en programas de EF para estudios probabilísticos de problemas de concreto armado y de interacción suelo-estructura. El primer autor ha trabajado en estas áreas (Tamayo y Awruch 2016a,b) y está dentro de los próximos planes incluir la metodología LAS en este tipo de problemas.

- La distancia de correlación es un parámetro de difícil de determinar en la práctica, pudiendo variar inclusive desde varios metros hasta kilómetros. En el abordaje de problemas de geotecnia se sugiere estudiar el efecto de este parámetro en la respuesta probabilística del sistema.

- La generación de campos estocásticos involucra actualmente la definición de un dominio paralelepípedo o rectangular, los cuales son representados por elementos cúbicos y rectángulos para el caso tridimensional y bidimensional, respectivamente. El estudio de problemas que involucran dominios curvos (reactores nucleares en estructuras de concreto armado, depósitos, etc.) requiere el establecimiento de una regla de mapeo adicional que permita convertir un dominio curvo en uno rectangular. Esta es una tarea a desarrollar en futuras pesquisas.

\section{REFERENCIAS}

[1] Bari, W. Modeling of ground improvement by vertical drains inhighly variable soils, $\mathrm{PhD}$ thesis. Curtin University, Western. 2012.

[2] Bressani, L.A., COSTA, E.A. Probabilistic evaluation of the stability of a slope colluvionar of basalt (In Portuguese), IV Cobrae-Conferencia Brasileira sobre Estabilidade de Encostas, COBRAE, Salvador, BA, Brazil, Vol. 4, p. 699-709, 2005.

[3] CHOK, Y. Modelling the effects of soil variability and vegetation on the stability of natural slopes, 2008. $\mathrm{PhD}$ thesis. School of Civil, Environmental and Mining Engineering, The Universityof Adelaida.

[4] Cavalcante, S.P.P.C., Vieira, V.P.P.B., Coutinho, R.Q. Stability risk analysis of downstream slope of an operating dam (In Portuguese), IV COBRAEConferencia Brasileira sobre Estabilidade de Encostas, COBRAE, Salvador, BA, Brazil, Vol. 4, p. 515-530, 2005.

[5] Fenton, G.A. Simulation and analysis of random fields generators, 1990. $\mathrm{PhD}$ thesis. Princeton University, USA.

[6] Fenton, G.A., Griffiths, D.V. Risk "Assessment in Geotechnical Engineering”, 1st ed., USA: John Wiley and Sons, 2008.

[7] Fenton, G.E., Vanmarcke, E. Simulation of random fields via local average subdivision. Journal of Engineering Mechanics, v. 116, p. 1733-1749, 1990.

[8] Lamus, Avelino Amado; Martínez, Edgar Hernán; Maldonado, Esperanza. Estimación del daño sísmico para puentes de varios vanos a partir de los modelos de Park y del ATC - 6.2. Revista UIS Ingenierías, [S.1.], v. 2, n. 1, ene. 2012. ISSN 2145-8456. 
[9] Lumb, P. The variability of natural soils. Canadian Geotechnical Journal, v. 3, p. 74-97, 1966.

[10] Paiboon, J. Numerical analysis of homogenization using random finite element method, 2013. PhD thesis. Colorado State University, Colorado.

[11] Smith, I. M., Griffiths, D.V., Margetts, L. Programming the finite element. John Wiley and Sons Ltd, United Kingdom, 2014.

[12] Tamayo, J.P.L., Awruch, A.M. On the validation of a numerical model for the analysis of soil-structure interaction problems. Latin American Journal of Solids and Structures, v. 13, n. 8, Jul - Dic, 2017. ISSN 1679-7825.

[13] Tamayo, J.P.L., Awruch, A.M. Numerical simulation of reinforced concrete nuclear containment under extreme loads. Structural Engineering and Mechanics, v. 58, n. 5, 2016b. ISSN 1225-4568.

[14] Vanmarcke, E. H. Random fields: analysis and synthesis. The MIT press, Cambridge, Massachusetts, USA, 1983.

[15] Vanmarcke, E. H. Probabilistic modelling of soil profiles. Journal of Geotechnical Engineering Division, v. 103, p. 1227-1246, 1977. 